\title{
The impact of gravidity, symptomatology and timing of infection on placental malaria
}

\author{
Erin E. Tran ${ }^{1,2}$, Morgan L. Cheeks ${ }^{1}$, Abel Kakuru3 ${ }^{3}$, Mary K. Muhindo 3 , Paul Natureeba ${ }^{3}$, Miriam Nakalembe ${ }^{4}$, \\ John Ategeka ${ }^{3}$, Patience Nayebare ${ }^{3}$, Moses Kamya ${ }^{4}$, Diane Havlir ${ }^{5}$, Margaret E. Feeney ${ }^{6}$, Grant Dorsey ${ }^{5}$ \\ and Stephanie L. Gaw ${ }^{1 *}$ (1)
}

\begin{abstract}
Background: Placental malaria is associated with increased risk of adverse perinatal outcomes. While primigravidity has been reported as a risk factor for placental malaria, little is known regarding the relationship between gravidity, symptomatology and timing of Plasmodium falciparum infection and the development of placental malaria.

Methods: The aim of this study was to investigate the relationship between the development of placental malaria and gravidity, timing of infection, and presence of symptoms. This is a secondary analysis of data from a double-blind randomized control trial of intermittent preventive therapy during pregnancy in Uganda. Women were enrolled from 12 to 20 weeks gestation and followed through delivery. Exposure to malaria parasites was defined as symptomatic (fever with positive blood smear) or asymptomatic (based on molecular detection of parasitaemia done routinely every 4 weeks). The primary outcome was placental malaria diagnosed by histopathology, placental blood smear, and/or placental blood loop-mediated isothermal amplification. Multivariate analyses were performed using logistic regression models. Subgroup analysis was performed based on the presence of symptomatic malaria, gravidity, and timing of infection.
\end{abstract}

Results: Of the 228 patients with documented maternal infection with malaria parasites during pregnancy, 101 (44.3\%) had placental malaria. Primigravidity was strongly associated with placental malaria (aOR 8.90, 95\% Cl 4.34$18.2, \mathrm{p}<0.001$ ), and each episode of malaria was associated with over a twofold increase in placental malaria (aOR $2.35,95 \% \mathrm{Cl} 1.69-3.26, \mathrm{p}<0.001)$. Among multigravid women, the odds of placental malaria increased by $14 \%$ with each advancing week of gestation at first documented infection ( $a \mathrm{OR} 1.14,95 \% \mathrm{Cl} 1.02-1.27, \mathrm{p}=0.02$ ). When stratified by the presence of symptoms, primigravidity was only associated with placental malaria in asymptomatic women, who had a 12-fold increase in the odds of placental malaria (aOR 12.19, 95\% Cl 5.23-28.43, p<0.001).

Conclusions: Total number of $P$. falciparum infections in pregnancy is a significant predictor of placental malaria. The importance of timing of infection on the development of placental malaria varies based on gravidity. In primigravidas, earlier asymptomatic infections were more frequently identified in those with placental malaria, whereas in multigravidas, parasitaemias detected later in gestation were associated with placental malaria. Earlier initiation of an effective intermittent preventive therapy may help to prevent placental malaria and improve birth outcomes, particularly in primigravid women.

\footnotetext{
*Correspondence: stephanie.gaw@ucsf.edu

${ }^{1}$ Division of Maternal Fetal Medicine, Department of Obstetrics Gynecology \& Reproductive Sciences, University of California San

Francisco, 513 Parnassus Ave. HSE16, Box 0556, San Francisco, CA 94143, USA

Full list of author information is available at the end of the article
}

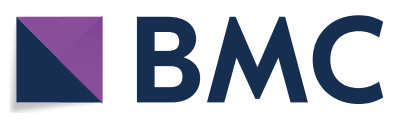

(c) The Author(s) 2020. This article is licensed under a Creative Commons Attribution 4.0 International License, which permits use, sharing, adaptation, distribution and reproduction in any medium or format, as long as you give appropriate credit to the original author(s) and the source, provide a link to the Creative Commons licence, and indicate if changes were made. The images or other third party material in this article are included in the article's Creative Commons licence, unless indicated otherwise in a credit line to the material. If material is not included in the article's Creative Commons licence and your intended use is not permitted by statutory regulation or exceeds the permitted use, you will need to obtain permission directly from the copyright holder. To view a copy of this licence, visit http://creativeco mmons.org/licenses/by/4.0/. The Creative Commons Public Domain Dedication waiver (http://creativecommons.org/publicdomain/ zero/1.0/) applies to the data made available in this article, unless otherwise stated in a credit line to the data. 
Keywords: Perinatal outcome, Infectious disease, Pregnancy, Global health, Primigravid, Africa, Obstetrics, Plasmodium falciparum

\section{Background}

Infection with malaria parasites during pregnancy is associated with increased risk of maternal and neonatal morbidity and mortality. In sub-Saharan Africa, where an estimated 11 million pregnancies are affected by malaria infection [1], malaria is thought to cause up to 100,000 infant deaths each year, the majority of which are secondary to prematurity, low birth weight, and neonatal anaemia [2, 3]. A unique characteristic of Plasmodium falciparum is the ability of infected erythrocytes to sequester in the placenta, leading to inflammation and adverse birth outcomes [4]. Placental malaria has been associated with increased risks of adverse obstetric outcomes including maternal anaemia, preterm delivery, fetal growth restriction, low birth weight, and maternal and neonatal mortality [5-7].

Previous studies have shown that primigravidity is a risk factor for placental malaria and resulting obstetric morbidity $[8,9]$. A prior analysis of this study cohort found that placental malaria was more likely in women with high malaria burden (defined as $\geq 2$ episodes of symptomatic malaria or $\geq 50 \%$ positive loop-mediated isothermal amplification (LAMP) samples) and was associated with increased rates of preterm birth and a trend towards higher rates of small for gestational age (SGA) neonates [10]. However, little is known about whether the effect of gravidity on placental malaria varies by timing and symptomatology of $P$. falciparum. A better understanding of how these factors influence placental infection in primigravid versus multigravid women may allow for more targeted public health interventions to reduce the prevalence of placental malaria and improve obstetric outcomes in both groups. Therefore, the main objective of this study was to assess whether the impact of gravidity on placental malaria varies by the presence of symptoms and/or timing of infection.

\section{Methods}

\section{Study procedures}

This was a secondary analysis of data collected from a double-blind, randomized trial of intermittent preventive therapy during pregnancy in Tororo, Uganda, a rural district in southeastern Uganda with an estimated entomologic inoculation rate of 310 infectious bites per person-year [11]. Participants were enrolled from June to October 2014; the complete study protocol for the parent study has been described elsewhere [11]. Briefly, pregnant women were enrolled at $12-20$ weeks gestation and randomized to preventive therapy with sulfadoxine-pyrimethamine given every 8 weeks, dihydroartemisinin-piperaquine given every 8 weeks, or dihydroartemisinin-piperaquine given every 4 weeks during pregnancy. All participants were at least 16 years of age and HIV-uninfected at the time of enrollment.

At study enrollment, participants were assessed with standardized history and physical exam including confirmation of gestational age (GA) by ultrasound. Routine visits were scheduled every 4 weeks and included collection of dried blood spots for loop-mediated isothermal amplification (LAMP). Study participant were encouraged to come the study clinic any time they were ill and if they presented with a documented fever (tympanic temperature $\geq 38.0{ }^{\circ} \mathrm{C}$ ) or a reported history of fever within $24 \mathrm{~h}$ prior to presentation, peripheral blood was collected for a thick blood smear. If the thick smear was positive, the participant was diagnosed with symptomatic malaria and treated with artemether-lumefantrine. Because LAMP results were not available until after completion of the trial, women with asymptomatic parasitaemia did not receive treatment. For the purposes of this analysis, women who presented with fever, but later had a positive LAMP were considered to have asymptomatic parasitaemia.

Placental tissue for histopathology, placental blood for thick smears and LAMP were collected within $1 \mathrm{~h}$ of delivery. Women who delivered at home were visited by study staff as soon as possible after delivery for evaluation and collection of specimens. Women with evidence of any infection with malaria parasites during pregnancy, known birth outcomes, and complete placental analysis (including placental histopathology, placental blood microscopy, and placental blood LAMP) were included in this study. Plasmodium falciparum infection was defined as both symptomatic malaria (fever and positive thick smear) and/or asymptomatic parasitaemia (peripheral maternal parasitaemia by LAMP in the absence of symptoms). The primary outcome, placental malaria, was defined as any evidence of placental infection, including the presence of parasites or pigment by histopathology, parasites detected in placental blood by microscopy, or positive placental blood LAMP.

\section{Laboratory methods}

Dried blood spot samples were tested for $P$. falciparum DNA using LAMP at enrollment and every 4 weeks through pregnancy, as previously described [12, 13]. 
Placental tissue samples were fixed with formalin and embedded in paraffin. They were then processed and examined by two independent readers using a standardized case record form for histological evidence of placental malaria [11]. Any discrepancy between the readers was resolved by a third reader. Blood smears were stained with $2 \%$ Giemsa and evaluated by two trained laboratory technicians. A smear was considered negative if no asexual parasites were detected in 100 high-powered fields. Any discrepancies were again resolved by a third reader.

\section{Clinical variables of interest}

Demographic data including maternal age, gravidity, body mass index (BMI), GA at enrollment and delivery, possession of bed net at enrollment, wealth status, and intermittent preventive therapy treatment arm were collected. Wealth status was defined as lowest, middle, and highest tertiles at study enrollment based on a composite variable of land ownership and possession of several household items [11]. GA was established at enrollment by obstetric dating by last menstrual period and ultrasound confirmation per WHO guidelines [14]. Malaria infection during pregnancy was defined as both symptomatic malaria and asymptomatic parasitaemia as previously mentioned, and longitudinal data on timing of infection during pregnancy were collected.

Birth outcomes including GA at delivery, birthweight, and fetal and neonatal survival to hospital discharge were collected and reported in previous analysis of this data set [10]. Preterm delivery was defined as delivery at $<37$ weeks GA. SGA was defined as birthweight $<10 \%$ ile for GA based on East African fetal weight standards [15] which have been shown to be more accurate than other international growth standards in diagnosing SGA in this population [10, 16, 17]. For twin gestations, outcomes were considered positive if the outcome was seen in at least one twin or placenta.

\section{Statistical methods}

Data analyses were performed using Stata 14 (Stata Corp, College Station, TX). Wilcoxon Rank Sum was used to compare median values of continuous variables, all of which were non-normally distributed. Chi squared and Fisher exact tests were used to compare proportions. Multivariate binary logistic regression using placental malaria as the main outcome were performed and were adjusted for primigravidity, GA at study enrollment, and GA at the time of the first recorded infection in pregnancy. Variables were included in the multivariate model if they were significant in the univariate analysis. Multivariate analyses stratified by gravidity, symptomatology and GA at initial infection were performed to examine interactions between possible predictors. The decision to do stratified analyses was made based on the a priori hypothesis that the relationship between these predictors could not be fully explained by multivariate analysis alone. All $p$ values were two-sided and a value of $p<0.05$ was considered statistically significant.

\section{Results \\ Study participants}

Of the 300 HIV-negative women enrolled in the parent trial, 289 (96.3\%) were followed to delivery. Of these, nine (3.1\%) were excluded for incomplete analyses for placental malaria including seven who did not have placental biopsies taken for histopathology and an additional two with missing results for placental blood analyses. Five women with placental malaria did not have documented evidence of parasitaemia prior to delivery and, given the inability to determine timing or number of infections, they were excluded from the analysis. Of the remaining 275 women, 228 (82.9\%) had evidence of infection with malaria parasites during pregnancy and were included in the analyses. Of these, $127 / 228$ (55.7\%) did not have evidence of placental malaria and 101/228 (44.3\%) had placental malaria (Fig. 1). The number of participants diagnosed with placental malaria via each of the three methods used are displayed in Fig. 2. Histopathology, which also detects past placental malaria, was the most sensitive of the three methods.

The median age of study participants was 21.1 years, and 38.2 percent were primigravid. Demographic characteristics and obstetrical outcomes of the study participants stratified by placental malaria status are presented in Table 1 . Women with placental malaria were younger (19.0 years $(17.9-21.3)$ vs. $22.7(19.9-25.7) ; \mathrm{p}<0.01)$ (median (IQR)) and more likely to be primigravid (63.4\% vs. $18.1 \%, \mathrm{p}<0.01)$. Those with placental malaria also enrolled (and therefore initiated intermittent preventive therapy) at a slightly later GA (15.3 weeks (14.0-17.6) vs. $14.7(13.6-15.9)$ weeks, $\mathrm{p}<0.01)$ and were significantly more likely to have received sulfadoxine-pyrimethamine treatment as compared to dihydroartemisinin-piperaquine $(48.5 \%$ versus $31.5 \%, \mathrm{p} \leq 0.01)$. All of the included twin gestations $(n=4)$ had evidence of placental malaria in at least one placenta (Table 1). As previously reported [10], women with placental malaria delivered at an earlier GA (39.3 weeks (38.0-40.3) vs. $40.0(39.0-40.9), \mathrm{p}<0.01)$ and were more likely to deliver preterm $(<37$ weeks GA) $(15.0 \%$ vs. $4.7 \%, \mathrm{p}<0.01)$ with correspondingly low birth weight (Table 1). Additionally, infants from pregnancies affected by placental malaria were more likely to be SGA $(25.7 \%$ vs. $15.0 \%, p=0.04)$. There were no significant differences in fetal or neonatal survival between the groups. 


\section{6 women screened}

86 excluded during screening

36 prior SP or antimalarial therapy during pregnancy

25 gestational age greater than 20 weeks

11 HIV positive

3 intention of moving outside of study area

2 gestational age less than 12 weeks

2 chronic medical condition

2 less than 16 years of age

1 non-viable pregnancy

1 early or active labor

1 residence greater than $30 \mathrm{~km}$ from clinic

1 does not plan to deliver in the hospital

1 unwilling to avoid medications outside protocol

300 women delivered

11 withdrawn before delivery

5 unable to locate for $>60$ days

5 moved out of study area

1 became HIV-infected

47 no malaria diagnosed during pregnancy and all samples negative for malaria parasites by LAMP

5 diagnosed with placental malaria but no prior infection

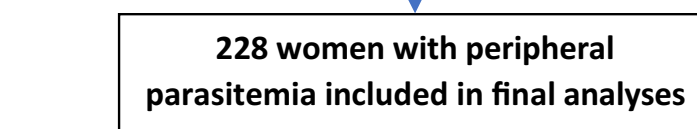

$\nabla$

No Placental Malaria $(n=127)$

Symptomatic or asymptomatic infection with confirmatory blood smear or laboratory evidence of malaria parasitemia by LAMP and no evidence of placental malaria

289 followed to delivery

(1)
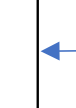


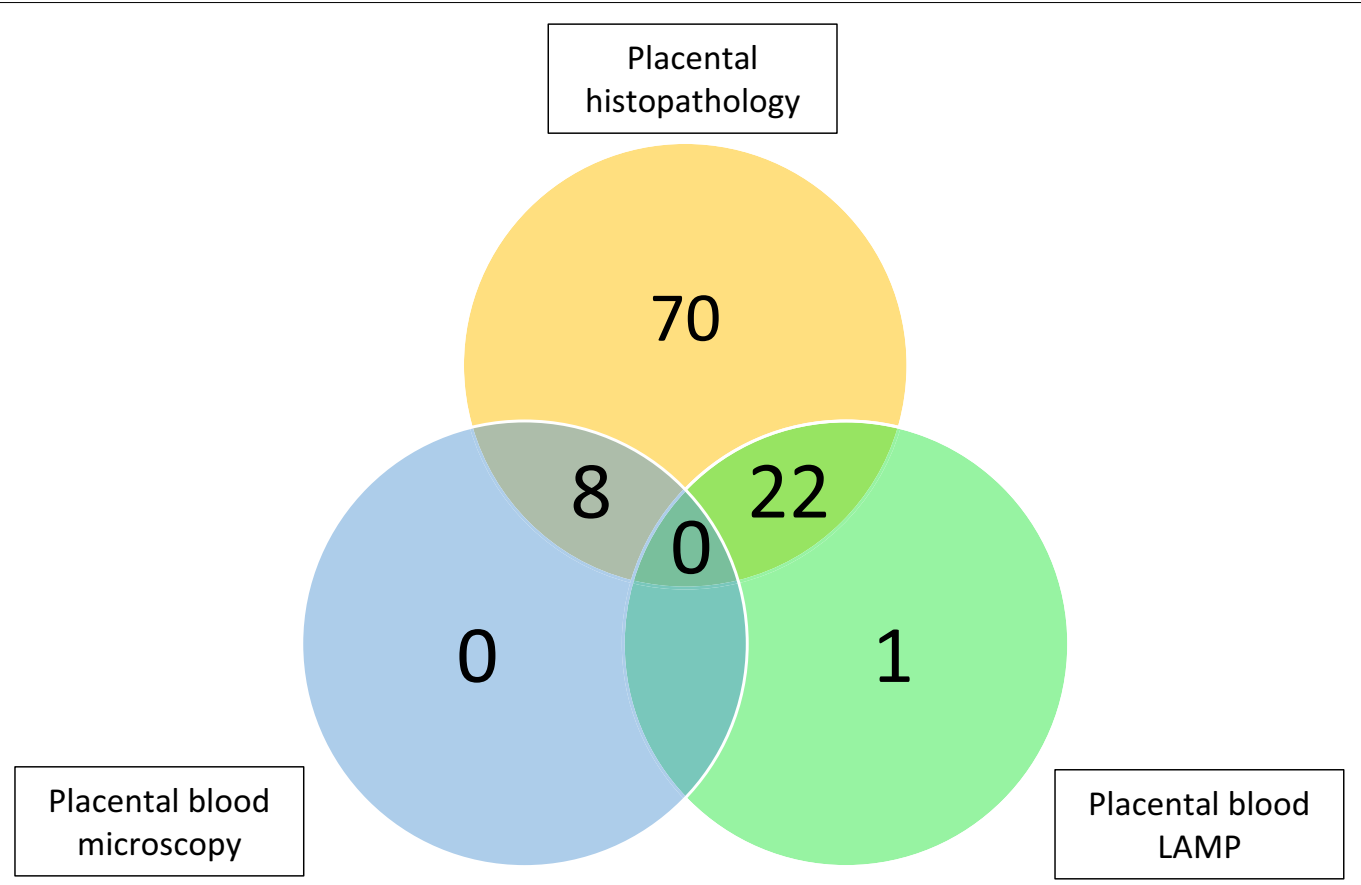

Fig. 2 Number of placental malaria infections diagnosed by each method. The yellow circle holds women diagnosed by placental histopathology. The blue circle indicates diagnosis via placental blood microscopy, and the green circle represents diagnosis via placental blood LAMP

\section{Longitudinal measures of $P$. falciparum infection}

Timing of initial documented infection and first episode of symptomatic malaria in women with and without placental malaria are depicted graphically in Fig. 3. Characteristics of infection are compared by placental malaria status in Table 2. Any symptomatic malaria at any time during pregnancy was more common in patients with placental malaria than those without placental malaria (29.7\% vs. $18.1 \%, p=0.04)$. Of those with at least one episode of symptomatic malaria, those with placental malaria had their first episode of symptomatic malaria at a later GA as compared to those without placental malaria (24.0 weeks (18.9-28.3) vs. 18.3 (16.1-20.0), $\mathrm{p} \leq 0.01$ ). GA at first documented infection (including symptomatic and asymptomatic) was not statistically different between the two groups $(p=0.15)$ (Table 2). Primigravidity, gestational age at enrollment, any symptomatic malaria during pregnancy and total number of times parasitaemia was detected in pregnancy were all controlled for in multivariate analyses because they were significant in univariate analyses. Gestational age at first documented infection was included in multivariate modeling rather than GA at first symptomatic infection to allow for examination of timing of infection without overfitting for symptomatology. In multivariate analyses, primigravidity, later GA at enrollment and increasing frequency of infections detected during pregnancy remained strongly associated with development of placental malaria (aOR 9.06,
95\% CI 4.38-18.71, p<0.001; aOR 1.24, 95\% CI 1.02$1.50, \mathrm{p}=0.03 ;$ aOR 2.21, 95\% CI 1.67-2.93, $\mathrm{p}<0.001$, respectively) (Table 3 ). As primigravid women are at increased risk of malaria infection, we also conducted the multivariate analysis without controlling for total number of infections in pregnancy in the model (Additional file 1: Table S1). Overall trends and statistical significance of findings for primigravidity and gestational age at enrollment were unchanged, and GA of first documented infection remains statistically insignificant. Any symptomatic malaria during pregnancy became statistically significant.

\section{Subgroup analyses}

In primigravidas, each additional week of GA at enrollment was associated with an increase in the odds of placental malaria (aOR 1.84, 95\% CI 1.21-2.78, p <0.01), but this association was not seen in multigravidas. In multigravid women, each additional week of gestation at initial detected infection was associated with a 1.12-fold increase in odds of placental malaria (aOR 1.12, 95\% CI $1.02-1.24, \mathrm{p}=0.02$ ). This trend was not seen in primigravidas. Total number of times infection was detected in pregnancy remained strongly associated with placental malaria in both primigravid and multigravid women (Table 4 and Fig. 4).

Multivariate analyses stratified by presence of any symptomatic malaria or only asymptomatic parasitaemia 
Table 1 Characteristics of study participants by placental malaria status

\begin{tabular}{|c|c|c|c|}
\hline \multirow[t]{2}{*}{ Variable } & \multicolumn{3}{|c|}{ Placental malaria status in pregnancy } \\
\hline & No placental malaria $(n=127)$ & Placental malaria $(n=101)$ & $p$ value \\
\hline Maternal age, years & $22.7(19.9-25.7)$ & $19.0(17.9-21.3)$ & $<0.001$ \\
\hline Primigravid & $23(18.1)$ & $64(63.4)$ & $<0.001$ \\
\hline $\mathrm{BMI}, \mathrm{kg} / \mathrm{m}^{2}$ & $20.6(19.0-22.2)$ & $21.0(19.7-22.7)$ & 0.08 \\
\hline Twin gestation ${ }^{\mathrm{a}}$ & $0(0.0)$ & $4(4.0)$ & 0.04 \\
\hline GA at enrollment, weeks & $14.7(13.6-15.9)$ & $15.3(14.0-17.6)$ & 0.003 \\
\hline Household wealth index & & & 0.80 \\
\hline Lowest tertile & $46(36.2)$ & 37 (36.6) & \\
\hline Middle tertile & $42(33.1)$ & $37(36.6)$ & \\
\hline Highest tertile & $39(30.7)$ & $27(26.7)$ & \\
\hline Intermittent preventive therapy drug & & & 0.01 \\
\hline Sulfadoxine-pyrimethamine & $40(31.5)$ & $49(48.5) \mathrm{s}$ & \\
\hline Dihydroartemisinin-piperaquine & $87(68.5)$ & $52(51.5)$ & \\
\hline GA at delivery, weeks & $40.0(39.0-40.9)$ & $39.3(38.0-40.3)$ & $<0.001$ \\
\hline Preterm birth < 37 weeks GA & $6(4.7)$ & $15(14.9)$ & 0.01 \\
\hline Very preterm birth $<32$ weeks GA & $1(0.8)$ & $4(4.0)$ & 0.17 \\
\hline Low birth weight ${ }^{\mathrm{b}}$ & $10(7.9)$ & $19(18.8)$ & 0.01 \\
\hline Small for gestational age $\mathrm{e}^{c}$ & $19(15.0)$ & $26(25.7)$ & 0.04 \\
\hline Stillbirth & $0(0.0)$ & $2(2.0)$ & 0.09 \\
\hline Neonatal demise ${ }^{d}(N=224)$ & $4(3.2)$ & $0(0.0)$ & 0.13 \\
\hline
\end{tabular}

$B M I$ body mass index, GA gestational age, $S G A$ small for gestational age

Statistically significant $\mathrm{p}$ values $(<0.05)$ are indicated in italics

a Information missing for 1 patient

b Defined as birthweight $<2500 \mathrm{~g}$

c Defined as birthweight $<10 \%$ ile for GA based on East African growth standards. ${ }^{15}$

${ }^{d}$ Data unavailable for four patients including two in the peripheral malaria group and 2 in the placental malaria group

Data are presented as median (interquartile range) or $\mathrm{n}(\%)$. Wilcoxon Rank Sum and Chi squared or Fischer Exact tests were used to compare nonparametric continuous variables and proportions, respectively. Pregnancies with peripheral malaria only (without placental malaria) and pregnancies with placental malaria are the comparison groups for the $\mathrm{p}$ values

was also performed. In women with only asymptomatic parasitaemia, primigravidity was associated with over a 12 -fold increase in odds of developing placental malaria (aOR 12.07, 95\% CI 5.24-27.81, $\mathrm{p}<0.001$ ), while there was no statistically significant association between primigravidity and placental malaria among those with any symptomatic malaria. In both groups, total number of infections in pregnancy remained highly associated with placental malaria (Table 5).

Lastly, multivariate analyses were stratified by GA at initial documented infection. These categories were selected based on timing of enrollment in the study and subsequent testing: $<14$ weeks (enrollment in first trimester), 14-19.9 weeks (enrollment in second trimester), and $\geq 20$ weeks gestation (infection after study enrollment). Primigravidity was strongly associated with placental malaria in women whose initial documented infection occurred prior to 14 weeks (aOR 29.30, 95\% CI $3.95-217.5, \mathrm{p}=0.001$ ), as well as in women whose first infection occurred between 14 and 19.9 weeks
(aOR 10.63, 95\% CI 4.12-27.42, $\mathrm{p}<0.001$ ). Primigravidity was not a significant predictor of placental malaria in women whose initial infection occurred at or beyond 20 weeks gestation. Total number of times infection was detected in pregnancy, however, was strongly associated with placental malaria in women whose first infection occurred at or beyond 20 weeks (aOR 5.61, 95\% CI 1.59$19.78, \mathrm{p}<0.01)$ as well as in women whose first infection occurred between 14 and 20 weeks gestation (aOR 2.63, 95\% CI 1.80-3.87, p<0.001) (Table 6).

\section{Discussion \\ Principal findings}

This study finds that the impact of gravidity on development of placental malaria varies by symptomatology and timing of $P$. falciparum infection. In women with only asymptomatic parasitaemia, primigravidity was the strongest predictor of placental malaria. In contrast, among women with symptomatic malaria, placental malaria was not significantly associated with 


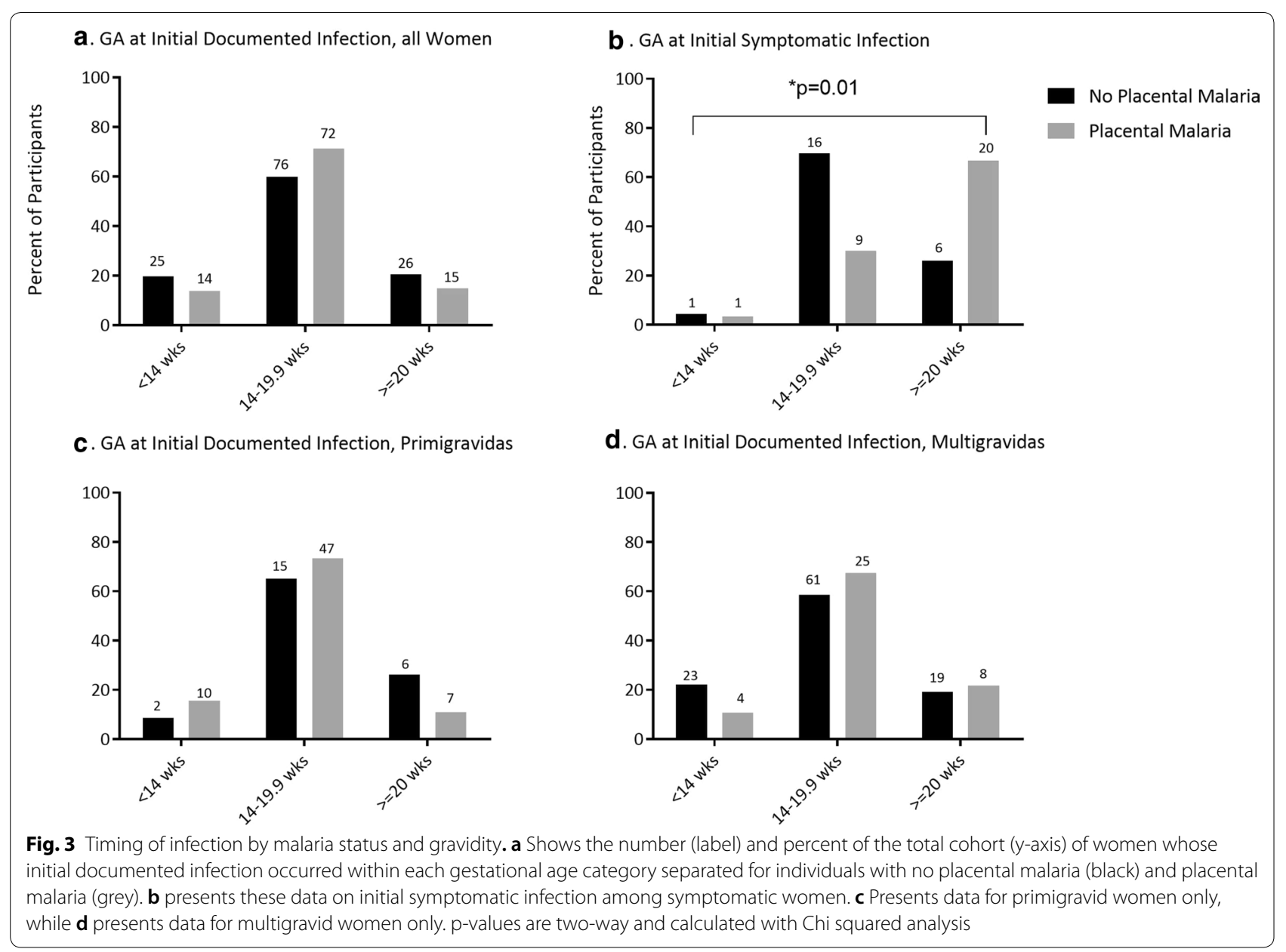

Table 2 Measures of malaria infection, by placental malaria status

\begin{tabular}{|c|c|c|c|}
\hline \multirow[t]{2}{*}{ Variable } & \multicolumn{3}{|c|}{ Placental malaria status in pregnancy } \\
\hline & No placental malaria $(n=127)$ & Placental malaria $(n=101)$ & $\mathrm{p}$ value \\
\hline Positive LAMP at enrollment ${ }^{a}$ & $84(66.1)$ & $76(75.3)$ & 0.14 \\
\hline Number of times parasitaemia detected per pregnancy & $2.0(2.0-3.0)$ & $3.0(2.0-5.0)$ & $<0.001$ \\
\hline Any symptomatic malaria during pregnancy & $23(18.1)$ & $30(29.7)$ & 0.04 \\
\hline GA at first detection of symptomatic malaria, weeks & $\begin{array}{l}N=23 \\
18.3(16.1-20.0)\end{array}$ & $\begin{array}{l}N=30 \\
24.0(18.9-28.3)\end{array}$ & 0.01 \\
\hline GA at initial documented parasitaemia, weeks & $15.9(14.3-17.9)$ & $16.1(14.7-18.6)$ & 0.15 \\
\hline
\end{tabular}

GA gestational age, LAMP loop mediated isothermal amplification

Data are presented as median (interquartile range) or $\mathrm{n}$ (\%). Wilcoxon Rank Sum and Chi squared tests were used to compare nonparametric continuous variables and proportions, respectively

Statistically significant $p$ values $(<0.05)$ are indicated in italics

a No patients had symptomatic malaria at the time of enrollment

gravidity. In regards to timing of infection, the magnitude of the association between primigravidity and the odds of placental malaria increased the earlier infection with malaria parasites was first detected, while multigravidity was associated with higher odds of placental malaria when infection occurred later in pregnancy. Of note, because the study population is women infected with malaria in pregnancy, the odds ratios 
Table 3 Predictors of placental malaria

\begin{tabular}{|c|c|c|c|}
\hline Variable & aOR & $95 \% \mathrm{Cl}$ & p value \\
\hline Primigravidity & 9.06 & $4.39-18.71$ & $<0.001$ \\
\hline GA at enrollment, weeks & 1.24 & $1.02-1.50$ & 0.03 \\
\hline $\begin{array}{l}\text { Any symptomatic malaria during preg- } \\
\text { nancy }\end{array}$ & 1.88 & $0.82-4.32$ & 0.14 \\
\hline GA of first documented infection, weeks & 1.06 & $0.97-1.16$ & 0.19 \\
\hline $\begin{array}{l}\text { Total number of times parasitaemia } \\
\text { detected in pregnancy }\end{array}$ & 2.21 & $1.67-2.93$ & $<0.001$ \\
\hline
\end{tabular}

\section{GA gestational age}

Statistically significant $p$ values $(<0.05)$ are indicated in italics

Multivariate binary logistic regression using placental malaria as the main outcome were performed and were adjusted for primigravidity, GA at study enrollment, any symptomatic malaria during pregnancy, GA of first documented infection, and total number of malaria infections (symptomatic and/or asymptomatic) in pregnancy. Variables were kept continuous where possible

reported in this study do not reflect odds of placental malaria in the general population, but rather odds of developing placental malaria if infected with $P$. falciparum in pregnancy.

When stratified by maternal gravidity, there were differences in the impact of timing of initial $P$. falciparum infection on the development of placental malaria. In multigravid women, later GA at initial infection is more strongly associated with placental malaria, while this is not observed in primigravid women. This suggests that much of the increased risk for primigravid women lies in early infection, which is not the case in multigravidas. This is consistent with the finding that later enrollment (and initiation of intermittent preventive therapy) increased odds of placental malaria in primigravid women but not in multigravid women.

This is the first study to explore the relationships between gravidity, symptomatology and timing of infection and the development of placental malaria. Prior literature on how the presence of malaria symptoms during pregnancy affects development of placental malaria is sparse. However, one cross sectional study of nulliparous Nigerian women found that more than $60 \%$ of asymptomatic women had evidence of placental parasitaemia at delivery, demonstrating that even asymptomatic infection has the potential to significantly impact obstetric outcomes [18]. This study adds to the current literature by highlighting that primigravid women are particularly vulnerable to placental malaria even when malaria infection remains clinically asymptomatic.

Table 4 Predictors of placental malaria stratified by gravidity

\begin{tabular}{|c|c|c|c|c|c|c|}
\hline \multirow[t]{2}{*}{ Variable } & \multicolumn{3}{|c|}{ Primigravid $(\mathrm{N}=87)$} & \multicolumn{3}{|c|}{ Multigravid (N=141) } \\
\hline & aOR & $95 \% \mathrm{Cl}$ & p value & aOR & $95 \% \mathrm{Cl}$ & p value \\
\hline GA at enrollment, weeks & 1.84 & $1.21-2.78$ & $<0.01$ & 1.12 & $0.88-1.44$ & 0.36 \\
\hline Any symptomatic malaria in pregnancy & 0.57 & $0.14-2.38$ & 0.44 & 2.89 & $1.05-7.95$ & 0.04 \\
\hline GA of first documented infection, weeks & 0.82 & $0.67-1.01$ & 0.06 & 1.12 & $1.02-1.24$ & 0.02 \\
\hline $\begin{array}{l}\text { Total number of times parasitaemia detected in } \\
\text { pregnancy }\end{array}$ & 2.70 & $1.44-5.06$ & $<0.01$ & 2.30 & $1.65-3.23$ & $<0.001$ \\
\hline
\end{tabular}

GA gestational age

Statistically significant $p$ values $(<0.05)$ are indicated in italics

Multivariate binary logistic regression using placental malaria as the main outcome were performed and were adjusted for GA at study enrollment, GA of first documented infection, and total number of malaria infections (symptomatic and/or asymptomatic) in pregnancy. Variables were kept continuous where possible
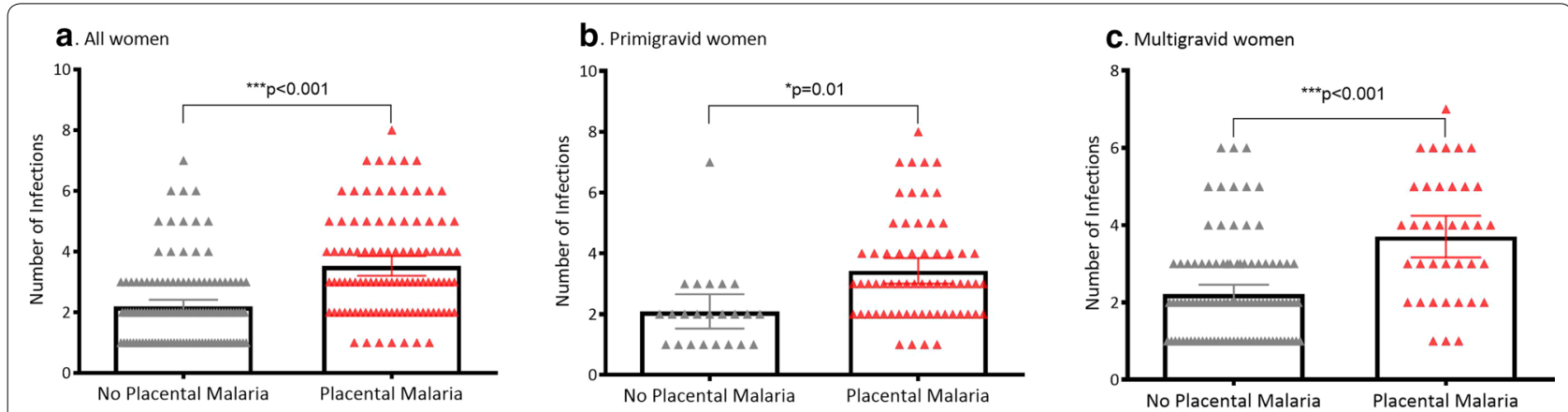

Fig. 4 Frequency of infection by malaria status and gravidity. a Shows the frequency of malaria infection by malaria status (no placental malaria versus placental malaria) for all women. $\mathbf{b}$ Shows these data for primigravid women, while $\mathbf{c}$ shows these data for multigravida women. Each triangle represents one woman. Mean and $95 \% \mathrm{Cl}$ are represented with error bars. p values are two-way and calculated with Chi squared analysis 
Table 5 Predictors of placental malaria stratified by presence of symptoms

\begin{tabular}{|c|c|c|c|c|c|c|}
\hline \multirow[t]{2}{*}{ Variable } & \multicolumn{3}{|c|}{ Any symptomatic Malaria $(\mathrm{N}=53)$} & \multicolumn{3}{|c|}{ Only asymptomatic parasitemia $(\mathrm{N}=175)$} \\
\hline & aOR & $95 \% \mathrm{Cl}$ & p value & aOR & $95 \% \mathrm{Cl}$ & p value \\
\hline Primigravid & 2.63 & $0.54-12.76$ & 0.23 & 12.07 & $5.24-27.81$ & $<0.001$ \\
\hline GA at enrollment, weeks & 1.36 & $0.89-2.08$ & 0.15 & 1.26 & $1.00-1.58$ & 0.05 \\
\hline GA of first documented infection, weeks & 1.13 & $0.99-1.23$ & 0.07 & 1.01 & $0.90-1.13$ & 0.83 \\
\hline $\begin{array}{l}\text { Total number of times parasitemia detected in } \\
\text { pregnancy }\end{array}$ & 2.43 & $1.37-4.30$ & $<0.01$ & 2.13 & $1.53-2.97$ & $<0.001$ \\
\hline
\end{tabular}

GA gestational age

Statistically significant $p$ values $(<0.05)$ are indicated in italics

Multivariate binary logistic regression using placental malaria as the main outcome were performed and were adjusted for GA at study enrollment, GA of first documented infection, and total number of malaria infections (symptomatic and/or asymptomatic) in pregnancy. Variables were kept continuous where possible

Table 6 Predictors of placental malaria stratified by gestational age at initial documented infection

\begin{tabular}{|c|c|c|c|c|c|c|c|c|c|}
\hline \multirow[t]{2}{*}{ Variable } & \multicolumn{3}{|c|}{$<14$ weeks $(\mathrm{N}=39)$} & \multicolumn{3}{|c|}{ 14-19.9 weeks $(N=148)$} & \multicolumn{3}{|c|}{$>=20$ weeks $(\mathrm{N}=41)$} \\
\hline & $\mathrm{aOR}$ & $95 \% \mathrm{Cl}$ & p value & aOR & $95 \% \mathrm{Cl}$ & p value & aOR & $95 \% \mathrm{Cl}$ & $p$ value \\
\hline Primigravid & 29.30 & $3.95-217.5$ & 0.001 & 10.63 & $4.12-27.42$ & $<0.001$ & 6.02 & $0.79-46.10$ & 0.08 \\
\hline GA at enrollment, weeks & 0.78 & $0.14-4.33$ & 0.78 & 1.58 & $1.19-2.09$ & 0.002 & 1.14 & $0.77-1.67$ & 0.51 \\
\hline Any symptomatic malaria in pregnancy & 1.44 & $0.12-16.71$ & 0.77 & 1.92 & $0.67-5.52$ & 0.23 & 9.90 & $0.96-102.48$ & 0.06 \\
\hline $\begin{array}{l}\text { Total number of times parasitaemia } \\
\text { detected in pregnancy }\end{array}$ & 1.22 & $0.65-2.30$ & 0.54 & 2.63 & $1.80-3.87$ & $<0.001$ & 5.61 & $1.59-19.78$ & $<0.01$ \\
\hline
\end{tabular}

GA gestational age

Statistically significant $\mathrm{p}$ values $(<0.05)$ are indicated in italics

Multivariate binary logistic regression using placental malaria as the main outcome were performed and were adjusted for GA at study enrollment, GA of first documented infection, and total number of malaria infections (symptomatic and/or asymptomatic) in pregnancy. Variables were kept continuous where possible

\section{Clinical implications}

Previous studies exploring the impact of timing of malaria infection during pregnancy have been mixed, and few prior studies have examined the relationship between timing of infection in pregnancy and development of placental malaria despite the well-established association of placental malaria with increased adverse obstetric outcomes $[10,19,20]$. Several studies have found increased rates of SGA [21], decreased birthweight [22, 23], and decreased fetal growth velocity [24] with early malaria infection while others have found more significant detrimental effects on fetal growth with infection in the second or third trimesters [25] and/or at delivery [26, 27].

Additionally, several studies found a negative impact on fetal growth with both early and late infection [28-31]. Malaria infection in the third trimester and/or at delivery has also been associated with increased risk of preterm birth $[21,30]$. Heterogeneity in the study designs, population demographics, surveillance protocols, diagnostic and treatment methodologies, and regional malaria characteristics may explain at least some of the discrepancies in these studies. Recently, an analysis of a separate cohort of women in Uganda found that burden of infection and timing of parasitaemia impact risk for placental malaria, which is consistent with the findings [32]. This study suggests that the importance of timing of infection on the development of placental malaria may vary based on gravidity. This finding may further explain why prior literature has painted an unclear picture of the impact of timing of infection on placental malaria.

Taken together, the results of this study have a number of implications for public health strategies going forward. Interventions aimed at preventing, identifying, and treating asymptomatic infections are likely needed to decrease placental malaria in primigravid women, whereas the magnitude of benefit in multigravid women may be less. Particular attention should be placed on preventing and treating early infections, both symptomatic malaria episodes as well as asymptomatic parasitaemia, in primigravid women. A challenge will be engaging women early in prenatal care. Finally, interventions aimed at reducing the overall number of $P$. falciparum infections during pregnancy are likely to benefit all pregnant women.

\section{Research implications}

It is important to note that the LAMP results were not known until after delivery, and so did not impact clinical care. Future studies investigating the impact of treating 
based on prenatal surveillance for asymptomatic infections may improve outcomes, particularly in primigravid women who are of highest risk for adverse outcomes. Also, the effect of parasite density during pregnancy cannot be evaluated with LAMP, which is reported as positive or negative detection of parasite nucleic acid in the sample. These are important areas of future study.

\section{Strengths and limitations}

There were many important strengths of this study including the longitudinal, prospective design, sonographically confirmed pregnancy dating, and rigorous screening protocol for both symptomatic and asymptomatic malaria infection throughout pregnancy. Additionally, examination of the placentas for evidence of malaria was undertaken by three different methods: placental blood smear, placental blood LAMP, and histopathology. Although the aforementioned strategy for placental analyses allowed for detection of microscopic as well as submicroscopic placental infection, histopathology was the most comprehensive method for diagnosis, as it also assessed for past placental malaria. Placental blood LAMP detected one additional case, but the overall benefit is unknown at this time.

There are several limitations of the study to acknowledge. Firstly, patients were enrolled between 12 and 20 weeks gestation, so details of malaria exposure in the first trimester are not known. The high proportion of patients with positive LAMP at enrollment, however, suggests widespread early exposure. Additionally, although monthly LAMP screening was performed throughout pregnancy, some malaria infections-particularly asymptomatic infections-that occurred between screening points may not have been detected. Our ability to distinguish persistent from recurrent infections as well as more definitively ascertain the timing of placental infection was also limited as parasite genotyping studies were not performed. Moreover, other obstetric comorbidities that may affect the rate of preterm birth and SGA, such as preeclampsia, bleeding or placental abruption, tobacco use, and infection were not consistently recorded.

\section{Conclusions}

Total number of $P$. falciparum infections in pregnancy is a significant predictor of placental malaria regardless of gravidity, symptomatology, or timing of $P$. falciparum infection in pregnancy. The influence of gravidity on development of placental malaria depends on symptomatology and timing of infection. Earlier initiation of an effective intermittent preventive therapy treatment may be needed to prevent placental malaria and improve birth outcomes, particularly in primigravid women.

\section{Supplementary information}

Supplementary information accompanies this paper at https://doi. org/10.1186/s12936-020-03297-3.

Additional file 1: Table S1. Multivariate analyses presented without controlling for total number of times parasitemia detected in pregnancy.

\section{Acknowledgements \\ Not applicable. \\ This data was presented in part at the 38th Annual Pregnancy Meeting for the Society for Maternal Fetal Medicine in Dallas, TX on January 29-February 3, 2018.}

\section{Authors' contributions}

SLG and EET conceived and designed the study. DVH, MRK, AK, MF and GD led the parent study clinical trial. MKM, PN, MN, JA, and PN participated in data collection. SLG, EET, MLC and GC participated in data analysis. SLG, EET and MLC drafted the first draft of the manuscript. All authors read and approved the final manuscript.

\section{Funding}

This study was supported by Eunice Kennedy Shriver National Institute of Child Health and Human Development (P01HD059454) and NIH/National Institute of Allergy and Infectious Diseases (K08Al141728-01 to SLG). Our funders played no role in the preparation of this manuscript.

\section{Availability of data and materials}

The datasets generated and/or analysed during the current study are not publicly available due to patient privacy concerns but are available from the corresponding author on reasonable request.

\section{Ethical approval}

All study participants provided informed consent. Ethical approval was received prior to study initiation from the Uganda National Council of Science and Technology (HS 1609 approved June 18, 2014), the Makerere University School of Biomedical Sciences Research and Ethics Committee (SBS 159 approved April 25, 2014), and the University of California, San Francisco Committee on Human Research (13-12743 approved February 6, 2014).

\section{Consent for publication}

No individual-level data are presented in this manuscript.

\section{Competing interests}

The authors report no conflict of interest.

\section{Author details}

${ }^{1}$ Division of Maternal Fetal Medicine, Department of Obstetrics Gynecology \& Reproductive Sciences, University of California San Francisco, 513 Parnassus Ave. HSE16, Box 0556, San Francisco, CA 94143, USA. ${ }^{2}$ Present Address: Division of Maternal Fetal Medicine, Department of Obstetrics \& Gynecology, NorthShore University HealthSystem, Evanston, IL, USA. ${ }^{3}$ Infectious Diseases Research Collaboration, Kampala, Uganda. ${ }^{4}$ Makerere University College of Health Sciences, Kampala, Uganda. ${ }^{5}$ Division of Infectious Diseases, Department of Medicine, University of California San Francisco, San Francisco, CA, USA. ${ }^{6}$ Division of Pediatric Infectious Diseases, Department of Pediatrics, University of California San Francisco, San Francisco, CA, USA.

Received: 14 April 2020 Accepted: 17 June 2020

Published online: 24 June 2020

\section{References}

1. WHO. World malaria report 2019. https://www.who.int/news-room/featu re-stories/detail/world-malaria-report-2019. Accessed 27 May 2020

2. Guyatt HL, Snow RW. The epidemiology and burden of Plasmodium falciparum-related anemia among pregnant women in sub-Saharan Africa. Am J Trop Med Hyg. 2001;64:36-44. 
3. Steketee RW, Nahlen BL, Parise ME, Menendez C. The burden of malaria in pregnancy in malaria-endemic areas. Am J Trop Med Hyg. 2001;64:36-44.

4. Miller LH, Baruch DI, Marsh K, Doumbo OK. The pathogenic basis of malaria. Nature. 2002;415:673-9.

5. Uneke CJ. Impact of placental Plasmodium falciparum malaria on pregnancy and perinatal outcome in sub-Saharan Africa: I: introduction to placental malaria. Yale J Biol Med. 2007;80:39-50.

6. Menendez C, Ordi J, Ismail MR, Ventura PJ, Aponte JJ, Kahigwa E, et al. The impact of placental malaria on gestational age and birth weight. J Infect Dis. 2000;181:1740-5.

7. Omer SA, Idress HE, Adam I, Abdelrahim M, Noureldein AN, Abdelrazig AM, et al. Placental malaria and its effect on pregnancy outcomes in Sudanese women from Blue Nile State. Malar J. 2017;16:374.

8. Lufele E, Umbers A, Ordi J, Ome-Kaius M, Wangnapi R, Unger H, et al. Risk factors and pregnancy outcomes associated with placental malaria in a prospective cohort of Papua New Guinean women. Malar J. 2017;16:427.

9. Ndeserua R, Juma A, Mosha D, Chilongola J. Risk factors for placental malaria and associated adverse pregnancy outcomes in Rufiji, Tanzania: a hospital based cross sectional study. Afr Health Sci. 2015;15:810-8.

10. Kapisi J, Kakuru A, Jagannathan P, Muhindo MK, Natureeba P, Awori P, et al. Relationships between infection with Plasmodium falciparum during pregnancy, measures of placental malaria, and adverse birth outcomes. Malar J. 2017;16:400

11. Kakuru A, Jagannathan $P$, Muhindo MK, Natureeba P, Awori P, Nakalembe $M$, et al. Dihydroartemisinin-piperaquine for the prevention of malaria in pregnancy. N Engl J Med. 2016;374:928-39.

12. Hopkins H, González IJ, Polley SD, Angutoko P, Ategeka J, Asiimwe C, et al. Highly sensitive detection of malaria parasitemia in a malaria-endemic setting: performance of a new loop-mediated isothermal amplification kit in a remote clinic in Uganda. J Infect Dis. 2013;208:645-52.

13. Rek J, Katrak S, Obasi H, Nayebare P, Katureebe A, Kakande E, et al. Characterizing microscopic and submicroscopic malaria parasitaemia at three sites with varied transmission intensity in Uganda. Malar J. 2016:15:470.

14. WHO. Manual of diagnostic ultrasound. Geneva, World Health Organization; 2013. https://apps.who.int/iris/handle/10665/85386. Accessed 18 May 2020

15. Schmiegelow C, Scheike T, Oesterholt M, Minja D, Pehrson C, Magistrado $P$, et al. Development of a fetal weight chart using serial trans-abdominal ultrasound in an East African population: a longitudinal observational study. PLoS ONE. 2012;7:e44773.

16. Stirnemann J, Villar J, Salomon LJ, Ohuma E, Ruyan P, Altman DG, et al. International estimated fetal weight standards of the INTERGROWTH-21st Project. Ultrasound Obstet Gynecol. 2017;49:478-86.

17. Kiserud T, Piaggio G, Carroli G, Widmer M, Carvalho J, Neerup Jensen L, et al. Correction: The World Health Organization fetal growth charts: a multinational longitudinal study of ultrasound biometric measurements and estimated fetal weight. PLoS Med. 2017;14:e1002284.

18. Bassey G, Nyengidiki TK, John CT. Prevalence of placenta Plasmodium parasitemia and pregnancy outcome in asymptomatic patients at delivery in a University Teaching Hospital in Nigeria. Niger J Clin Pract. 2015;18:27.

19. Rogerson SJ, Mwapasa V, Meshnick SR. Malaria in pregnancy: linking immunity and pathogenesis to prevention. Am J Trop Med Hyg. 2007;77:14-22.
20. Desai M, Phillips-Howard PA, Odhiambo FO, Katana A, Ouma P, Hamel MJ, et al. An analysis of pregnancy-related mortality in the KEMRI/CDC health and demographic surveillance system in western Kenya. PLoS ONE. 2013;8:e68733.

21. Moore KA, Simpson JA, Wiladphaingern J, Min AM, Pimanpanarak M, Paw $M K$, et al. Influence of the number and timing of malaria episodes during pregnancy on prematurity and small-for-gestational-age in an area of low transmission. BMC Med. 2017;15:117.

22. Valea I, Tinto H, Drabo MK, Huybregts L, Sorgho H, Ouedraogo J-B, et al. An analysis of timing and frequency of malaria infection during pregnancy in relation to the risk of low birth weight, anaemia and perinatal mortality in Burkina Faso. Malar J. 2012;11:71.

23. Machado Filho AC, da Costa EP, da Costa EP, Reis IS, Fernandes EAC, Paim BV, et al. Effects of vivax malaria acquired before 20 weeks of pregnancy on subsequent changes in fetal growth. Am J Trop Med Hyg. 2014;90:371-6.

24. Briand V, Saal J, Ghafari C, Huynh B-T, Fievet N, Schmiegelow C, et al. Fetal growth restriction is associated with malaria in pregnancy: a prospective longitudinal study in Benin. J Infect Dis. 2016;214:417-25.

25. Kalilani L, Mofolo I, Chaponda M, Rogerson SJ, Meshnick SR. The effect of timing and frequency of Plasmodium falciparum infection during pregnancy on the risk of low birth weight and maternal anemia. Trans $R$ Soc Trop Med Hyg. 2010;104:416-22.

26. Kalilani-Phiri L, Thesing PC, Nyirenda OM, Mawindo P, Madanitsa M, Membe $G$, et al. Timing of malaria infection during pregnancy has characteristic maternal, infant and placental outcomes. PLoS ONE. 2013;8:e74643.

27. Verhoeff FH, Brabin BJ, van Buuren S, Chimsuku L, Kazembe P, Wit JM, et al. An analysis of intra-uterine growth retardation in rural Malawi. Eur J Clin Nutr. 2001;55:682-9.

28. Cottrell G, Mary J-Y, Barro D, Cot M. The importance of the period of malarial infection during pregnancy on birth weight in tropical Africa. Am J Trop Med Hyg. 2007;76:849-54.

29. Huynh B-T, Fievet N, Gbaguidi G, Dechavanne S, Borgella S, Guézo-Mévo $B$, et al. Influence of the timing of malaria infection during pregnancy on birth weight and on maternal anemia in Benin. Am J Trop Med Hyg. 2011;85:214-20.

30. Sullivan AD, Nyirenda T, Cullinan T, Taylor T, Harlow SD, James SA, et al. Malaria infection during pregnancy: intrauterine growth retardation and preterm delivery in Malawi. J Infect Dis. 1999;179:1580-3.

31. McClure EM, Meshnick SR, Lazebnik N, Mungai P, King CL, Hudgens M, et al. A cohort study of Plasmodium falciparum malaria in pregnancy and associations with uteroplacental blood flow and fetal anthropometrics in Kenya. Int J Gynaecol Obstet. 2014;126:78-82.

32. Briggs J, Ategeka J, Kajubi R, Ochieng T, Kakuru A, Ssemanda C, et al. Impact of microscopic and submicroscopic parasitemia during pregnancy on placental malaria in a high-transmission setting in Uganda. J Infect Dis. 2019;220:457-66.

\section{Publisher's Note}

Springer Nature remains neutral with regard to jurisdictional claims in published maps and institutional affiliations.

Ready to submit your research? Choose BMC and benefit from

- fast, convenient online submission

- thorough peer review by experienced researchers in your field

- rapid publication on acceptance

- support for research data, including large and complex data types

- gold Open Access which fosters wider collaboration and increased citations

- maximum visibility for your research: over 100M website views per year

At $\mathrm{BMC}$, research is always in progress.

Learn more biomedcentral.com/submissions 